\title{
Beziehung zwischen IT-Erfolgsfaktoren und IT-Risikomanagement im eGovernment
}

IT-Erfolgsfaktoren beschreiben Bedingungen, die maßgeblich zur erfolgreichen Umsetzung von ITProjekten beitragen. Über das IT-Risikomanagement wird dabei präventiv versucht, kritischen Erfolgsfaktoren Rechnung zu tragen, was u.a. durch den Einsatz von IT-Governance-Methoden unterstützt und geprüft werden kann. Der vorliegende Beitrag setzt sich mit der Beziehung zwischen IT-Risikomanagement und IT-Erfolgsfaktoren im eGovernment und deren Zusammenhang auseinander. Dazu werden Erfolgsfaktoren aus acht Studien ausgewertet und konsolidiert. Es wird der Frage nachgegangen, inwieweit die identifizierten Erfolgsfaktoren in den in der schweizerischen Bundesverwaltung eingesetzten IT-Governance-Methoden im IT-Risikomanagement berücksichtigt werden. Aus der theoretischen Gegenüberstellung wird zusätzlicher Handlungsbedarf in der Methodenunterstützung für das Risikomanagement ersichtlich, um Risiken im eGovernment effektiv antizipieren zu können.

\section{Inhaltsübersicht}

1 Einleitung

1.1 Problemstellung

1.2 Fragestellungen

1.3 Methodisches Vorgehen

2 IT-Management in der öffentlichen Verwaltung

2.1 Charakterisierung von eGovernmentProjekten

2.2 Erfolg und Misserfolg

3 Synopse der Erfolgsfaktoren

3.1 Untersuchte Studien

3.2 Darstellung der Erfolgsfaktoren
4 Einsatz von IT-Governance-Methoden in der schweizerischen Bundesverwaltung

4.1 Beschreibung der IT-GovernanceMethoden

4.2 Vergleich der Methoden in Analogie zu den Erfolgsfaktoren

5 Herausforderungen für das

IT-Risikomanagement

6 Literatur

\section{Einleitung}

\subsection{Problemstellung}

Mit der zunehmenden IT-Unterstützung in der öffentlichen Verwaltung werden durch Neugestaltung und Automatisierung von Prozessen Effizienzziele (Qualität, Kosten und Zeit) im eGovernment verfolgt. Mit eGovernment als einem relevanten Bestandteil diverser Regierungs- und Verwaltungsreformprogramme wird versucht, Effektivitätssteigerungen im Verwaltungshandeln zu erzielen. Die Umsetzung dieser Programme wie auch die Erreichung der Ziele sind eng an das erfolgreiche und immer komplexere IT-Management gebunden. Die Implementierung von erfolgreichem eGovernment erfordert organisationale, prozessuale sowie kulturelle Veränderungen, die Anpassung oder Schaffung rechtlicher Grundlagen sowie technologische Innovationen. In der zunehmenden Komplexität des ITManagements, beispielsweise aufgrund wachsender Interoperabilitätsbedarfe und Vernetzung, mag ein Grund für das Risiko des häufigen Scheiterns von Projekten im eGovernment liegen. Die Zahlen sind in der Tat ernüchternd: 
Die Scheiterungsraten bewegen sich auf konstant hohem Niveau (vgl. [Mertens 2008]).

Forschung und Praxis untersuchen seit jeher empirisch das Phänomen des Scheiterns großer IT-Projekte, wie u.a. die in diesem Beitrag untersuchten Studien zeigen. Die in verschiedenen Untersuchungen abgeleiteten Erfolgsfaktoren und Heuristiken sollen zur Vermeidung von Fehlern und Risiken im IT-Management beitragen. Die daraus gewonnenen Erkenntnisse sollen - im Sinne des Risikomanagements - im IT-Management dazu dienen, das Scheitern von Projekten proaktiv zu verhindern. Vorgehensmodelle für das Projektmanagement und das Software Engineering sowie organisationale Funktionen wie das Qualitätsmanagement, das IT-Controlling und das IT-Auditing mögen Leitplanken bilden, um die Scheiterungsraten zu senken. Ganz zu minimieren oder gar zu eliminieren vermögen indes auch solche Ansätze das Risiko nicht. Von Interesse ist der Mechanismus des Scheiterns von eGovernment-Projekten vor dem Hintergrund der in einem spezifischen Projekt anwesenden Erfolgsfaktoren. Ein integriertes Risikomanagement, an dem es in Verwaltungen möglicherweise mangelt, hätte die Aufgabe, kritische Erfolgsfaktoren im dynamischen Management von Projekten und der IT proaktiv zu berücksichtigen. Daraus ergibt sich die für diesen Beitrag relevante Forschungslücke.

Es stellt sich aus Themensicht die Frage, ob und in welcher Form Managementkonzepte aus der Privatwirtschaft - und um ein solches handelt es sich mehrheitlich beim Thema (IT-) Risikomanagement - auf die öffentliche Verwaltung übertragen werden können. In der öffentlichen Verwaltung herrschen grundsätzlich andere Gegebenheiten als in der Privatwirtschaft. Der "Buchstabe des Gesetzes" und die Gleichheit der Bürger vor dem Staat dominieren das Verwaltungshandeln. Im Gegensatz dazu stehen der Wettbewerb und das gewinnorientierte Handeln in der freien Marktwirtschaft. Während das Risikomanagement in der Privatwirtschaft etwa auf die nachhaltige Erwirt- schaftung von Gewinnen ausgerichtet ist, müsste das Risikomanagement in der Verwaltung einerseits auf die Einhaltung der Rechtsgrundsätze und andererseits auf die Verhinderung der "Verschleuderung von Steuergeldern" ausgerichtet sein. Für das IT-Risikomanagement in der Verwaltung sollte die Erfüllung von Verwaltungsaufgaben im Vordergrund stehen, was operative Risiken der "Verwaltungsproduktion " sowie rechtliche und finanzielle Risiken im IT-Management umfasst. Das schweizerische Bundesgesetz über die Eidgenössische Finanzkontrolle (FKG) fordert die Vermeidung finanzieller Einbußen aufgrund gescheiterter Projekte sowie einen kosteneffizienten und wirkungsorientierten Einsatz finanzieller Mittel. Daraus lässt sich ein gesetzlicher Bedarf an das IT-Risikomanagement ableiten.

\subsection{Fragestellungen}

Im vorliegenden Beitrag stehen folgende Fragestellungen im Vordergrund: Welches sind eGovernment- oder IT-Erfolgsfaktoren im Management von IT-Projekten? Welche IT-GovernanceMethoden werden für das IT-Management der Verwaltung eingesetzt? Inwiefern korrespondiert der Einsatz dieser Methoden mit den Erfolgsfaktoren des IT-Managements? In welchen Zusammenhang können IT-Erfolgsfaktoren und das IT-Risikomanagement in der Verwaltung gestellt werden? Welche Rolle spielt das IT-Risikomanagement in den IT-Governance-Methoden der Verwaltung? Die Fragen werden teilweise anhand des Beispiels der schweizerischen Bundesverwaltung beantwortet.

\subsection{Methodisches Vorgehen}

Basierend auf einer Literaturrecherche wurden aus acht Studien mittels Inhaltsanalyse $30 \mathrm{Er}$ folgsfaktoren ausgearbeitet und in 15 Kategorien unterteilt. Im nachfolgenden Schritt wurden die Erfolgsfaktoren den in der schweizerischen Bundesverwaltung eingesetzten IT-Governance-Methoden gegenübergestellt. Davon ausgehend wird diskutiert, inwieweit die Er- 
folgsfaktoren in den erwähnten Methoden eine Berücksichtigung im IT-Risikomanagement erfahren.

\section{IT-Management in der öffentlichen Verwaltung}

In der öffentlichen Verwaltung kann von einem generischen IT-Managementmodell mit verwaltungsspezifischen Eigenheiten ausgegangen werden. Aus organisatorischer und prozessualer Sicht lässt sich das IT-Management in eine strategische Ebene (IT-Strategiemanagement und Führung) und eine operative prozessorientierte Ebene (Machbarkeitsstudien, Projekte, Betrieb) unterteilen [Moll 1994]. Für diesen Beitrag werden verschiedene Begriffe wie folgt verwendet: Unter IT-Management wird als übergeordneter Begriff die Aufbau- und Ablauforganisation der IT sowie die Konkretisierung strategischer IT-Aufgaben und die operativen Aufgaben der Planung, der Entwicklung sowie des Betriebs im Sinne des "Plan-Build-Run «-Ansatzes des IT-Lifecycles verstanden. Jeder Prozessbereich beinhaltet unterschiedliche Rollen, Funktionen und Aufgaben [Moll 1994]. Ausgehend von denselben lassen sich unterschiedliche Aspekte des IT-Risikomanagements adressieren. Die IT-Governance umfasst Methoden zur Sicherstellung eines aus betrieblicher Sicht effizienten Mitteleinsatzes im Bereich der IT. Dabei können IT-Governance-Methoden auf strategischer oder operativer Ebene zum Einsatz gelangen. Das IT-Management zeigt auf, wie etwas zu tun ist. Die IT-Governance zeigt auf, was zu tun ist. Erfolgsfaktoren, hier zusammenfassend bezeichnet für IT-Erfolgs- und -Misserfolgsfaktoren sowie IT-Risikofaktoren, sind Faktoren, die den Verlauf und/oder das Ergebnis von Aktivitäten im IT-Management maßgeblich beeinflussen. Ein Risiko bezeichnet die Unsicherheit eines Verlaufs/Ergebnisses in positiver wie in negativer Richtung. Als Risikomanagement wird ein systematischer Prozess zur Identifikation, Analyse und Kontrolle im Sinne von Überwachung und Steuerung von Risiken in Projekten oder Organisationen bezeichnet [Wallmüller 2004]. Ein umfassendes Risikomanagement im eGovernment erfordert eine organisatorische Verankerung entsprechend den strategischen und operativen IT-Rollen, -Funktionen und -Aufgaben. Generell gilt somit, dass das Management von Risiken in der Gesamtorganisation und nicht nur im IT-Bereich von Relevanz ist.

\subsection{Charakterisierung von eGovernment- Projekten}

eGovernment-Projekte werden im Kontext des öffentlichen Sektors abgewickelt. Dabei gilt es besondere Rahmenbedingungen zu beachten. So ist insbesondere auf die Komplexität von Projekten zu achten, die u.a. durch die Interoperabilität sowie die Multidisziplinarität gegeben ist. Zudem spielt die Projektgröße eine zentrale Rolle. So ist mit Laufzeiten von nicht selten mehr als 12 bis 18 Monaten zu rechnen, was eine erfolgreiche Abwicklung erschwert [POST 2003]. Aus den Fachmedien sind gelegentlich Berichte - mit entsprechender Publizität - über das Scheitern bzw. den Abbruch solcher Projekte zu entnehmen. Neuere Beispiele aus der Schweiz hierzu sind etwa die Projekte Simapz (eProcurement) und Insieme (eTaxation). Dem (IT-)Risikomanagement ist entsprechend wohl eine größere Beachtung zu schenken. Ein abgebrochenes oder frühzeitig beendetes Projekt ist nicht zwingend mit einem Misserfolg gleichzusetzen, wenn gute Gründe wie geänderte Rahmenbedingungen für einen Abbruch sprechen.

Insbesondere im politischen Umfeld von Verwaltungen können Änderungen der politischen Marschrichtung (z.B. nach Wahlen) Projekte auf unbestimmte Zeit stoppen, oder Projekte werden vor deren Fertigstellung beendet. Misserfolge bei dieser Größe von Projekten sind mit hohen Kosten verbunden. Unter anderem können Kosten von Misserfolgen durch präzise 
vertragliche Regelungen und durch ein integriertes Risikomanagement verhindert oder minimiert werden.

\subsection{Erfolg und Misserfolg}

Erfolgs- bzw. Misserfolgsraten von IT-Projekten gilt es genauer zu untersuchen. Die Messung des Erfolgs erweist sich als schwierig. Daher sind relevante und messbare Erfolgskriterien vor Beginn des Projektes klar zu definieren. Dennoch, Erfolg und Misserfolg können aus der Perspektive verschiedener Akteure unterschiedlich bewertet werden, weil subjektive Beurteilungen in unterschiedlichen Kontexten möglicherweise nicht vereinbar sind [Bronte-Stewart 2005]. Dies ergibt zwei zentrale Feststellungen: Die Beurteilung ist abhängig vom Beurteilungszeitpunkt und den Zielen bzw. Sichten, so etwa von der System-, Anwender-, Organisationsoder Strategiesicht [Peterson \& Kim 2000]; die Beurteilung sollte auf den mit dem Einsatz verbundenen Nutzen ausgerichtet werden und nicht nur einen isolierten Blick auf die einzelnen Phasen Plan, Build oder Run richten. Vielmehr ist zu beachten, was vor, während und nach der Umsetzung eines IT-Projektes auf der Geschäftsseite vorgeht. Besonderes Augenmerk ist aus der Sicht dieses Beitrags der Frage der Beziehung zwischen IT-Erfolgs- und -Risikofaktoren zu schenken. Erfolgsfaktoren können Risikofaktoren sein und umgekehrt. Entscheidend für den Unterschied ist die zeitliche Betrach- tungsperspektive. Die Beurteilung von Erfolgen geschieht in der Regel ex post. Die Beurteilung von Risken erfolgt in der Regel ex ante. Erfolge, ob negativ oder positiv, sind damit im Sinne des IT-Risikomanagements mit Eintretenswahrscheinlichkeiten versehen.

\section{Synopse der Erfolgsfaktoren}

Verschiedene Studien aus Wissenschaft und Praxis zeigen Erfolgsfaktoren auf, die den Verlauf und das Ergebnis von Aktivitäten im IT-Management beeinflussen können. Im Sinne von Best Practices sollten diese Faktoren im IT-Management berücksichtigt werden. Dies geschieht im Folgenden durch die Ausarbeitung von Erfolgsfaktoren aus acht Studien.

\subsection{Untersuchte Studien}

Für den Vergleich wurden acht Studien - wissenschaftliche Untersuchungen wie auch Empfehlungen und Berichte der öffentlichen Verwaltung - aus verschiedenen Bereichen herangezogen, um eine umfassende Identifikation und Kategorisierung von Erfolgsfaktoren anzustreben.

Kriterien für die Wahl der Studien waren ihr Bezug zur öffentlichen Verwaltung ( 5 von 8 Studien) und ihre Relevanz für bzw. ihre Verbreitung in der Praxis (3 von 8 Studien). Eine Charakterisierung der Studien erfolgt in Tabelle 1.

\begin{tabular}{|l|l|l|}
\hline Nr./Studie & Beschreibung & Methode und Wertung \\
\hline $\begin{array}{l}\text { 1: [Bronte-Stewart 2005]: } \\
\text { Developing a risk estimation } \\
\text { model from IT project failure } \\
\text { research }\end{array}$ & $\begin{array}{l}\text { Entwicklung eines Bewertungsmodells für Risiken in } \\
\text { IT-Projekten sowie Ableitung von Risikofaktoren aus } \\
\text { verschiedenen Studien }\end{array}$ & $\begin{array}{l}\text { Wissenschaftlich-theoretische Metastudie zu } \\
\text { Erfolgsfaktoren in IT-Projekten anhand von } \\
\text { Sekundäranalysen }\end{array}$ \\
\hline $\begin{array}{l}\text { 2: [OECD 2001]: The Hidden } \\
\text { Threat to E-Government }\end{array}$ & $\begin{array}{l}\text { Empfehlungen der OECD im Umgang mit IT-Projek- } \\
\text { ten in der öffentlichen Verwaltung }\end{array}$ & $\begin{array}{l}\text { Best Practices basierend auf Länderreports } \\
\text { undfentlichen Sektor aus 17 Ländern }\end{array}$ \\
\hline $\begin{array}{l}\text { 3: [Boehm 1991]: Software Risk } \\
\text { Management: Principles and } \\
\text { Practices }\end{array}$ & $\begin{array}{l}\text { Checkliste der zehn häufigsten Risiken in Software- } \\
\text { projekten sowie dazugehörige Risikomaßnahmen }\end{array}$ & $\begin{array}{l}\text { Praxis-Checkliste basierend auf einer } \\
\text { Umfrage bei erfahrenen Projektmanagern }\end{array}$ \\
\hline $\begin{array}{l}\text { 4: } \text { [Hall 1998]: Managing risk: } \\
\text { methods for software systems } \\
\text { development }\end{array}$ & $\begin{array}{l}\text { Beschreibung der zehn Standardrisiken von IT-Pro- } \\
\text { jekten der öffentlichen Verwaltung aus Sicht des } \\
\text { Kunden }\end{array}$ & $\begin{array}{l}\text { Durch formales Risiko-Assessment abgelei- } \\
\text { tete Standardrisiken von Projekten der } \\
\text { öffentlichen Verwaltung }\end{array}$ \\
\hline
\end{tabular}




\begin{tabular}{|l|l|l|}
\hline $\begin{array}{l}\text { 5: [Standish 1995]: CHAOS } \\
\text { Chronicles 1995 }\end{array}$ & $\begin{array}{l}\text { Erhebung der Erfolgs- und Misserfolgs-faktoren von } \\
\text { IT-Projekten und deren Erfolgsraten in verschiede- } \\
\text { nen Branchen }\end{array}$ & $\begin{array}{l}\text { Wiederkehrende Studie in Form von Umfra- } \\
\text { gen und Interviews mit IT-Managern (in } \\
\text { Fokusgruppen) in den USA }\end{array}$ \\
\hline $\begin{array}{l}\text { 6: [Heeks 2003]: eGovernment } \\
\text { for Development: Factor Model }\end{array}$ & $\begin{array}{l}\text { Modell von Erfolgs- und Fehlerfaktoren für eGovern- } \\
\text { ment in Entwicklungs- und Transitionsländern }\end{array}$ & $\begin{array}{l}\text { Empirische Ableitung von Erfolgsfaktoren } \\
\text { ausgehend von einer Reihe von Fallstudien }\end{array}$ \\
\hline $\begin{array}{l}\text { 7: [Evangelidis et al. 2002]: Risk } \\
\text { Assessment \& Success Factors } \\
\text { for eGovernment }\end{array}$ & $\begin{array}{l}\text { Beschreibung der Risikofaktoren im britischen } \\
\text { eGovernment sowie Klassifizie-rung derselben in die } \\
\text { Bereiche »Technological/Implementation«, "Social/ } \\
\text { Human«, "Security«, »Financial« und "Legal « }\end{array}$ & $\begin{array}{l}\text { Empirische Ausarbeitung der Entwicklungen } \\
\text { schen eGovernment }\end{array}$ \\
\hline $\begin{array}{l}\text { 8: [POST 2003]: } \\
\text { Government IT projects }\end{array}$ & $\begin{array}{l}\text { Analyse des Parliamentary Office of Science and } \\
\text { Technology von IT-Projekten/-Programmen in der } \\
\text { britischen Verwaltung; Beschreibung und Evalua- } \\
\text { tion von Maßnahmen zur Steigerung der Erfolgsra- } \\
\text { ten von IT-Projekten }\end{array}$ & $\begin{array}{l}\text { Analytisch-praktischer Bericht und Evalua- } \\
\text { tion von getroffenen Initiativen durch eine } \\
\text { Stelle der britischen Parlamentsdienste }\end{array}$ \\
\hline
\end{tabular}

Tab. 1: Untersuchte Studien

\subsection{Darstellung der Erfolgsfaktoren}

Die aus den acht Studien abgeleiteten Kategorien und extrahierten Erfolgsfaktoren sowie deren Beschreibung sind in Tabelle 2 dargestellt. Aus Tabelle 3, linker Teil, lässt sich schließen, aus welchen Studien die einzelnen Erfolgsfaktoren extrahiert wurden. Die Unterteilung der $30 \mathrm{Er}-$ folgsfaktoren in 15 Kategorien erfolgte mittels einer Inhaltsanalyse. Die Darstellung gibt ein zusammenfassendes Bild der in den studien identifizierten Erfolgsfaktoren wieder. Allerdings waren sogenannte weiche Faktoren in den untersuchten Studien lediglich vereinzelt oder indirekt vertreten, weshalb sie nicht in die Zusammenstellung aufgenommen wurden. Entsprechende weiche Erfolgsfaktoren wie Soft Skills oder psychologische Faktoren (Motivation, Vertrauen etc.) beeinflussen den Erfolg von Projekten ebenfalls stark und sind ebenso zu berücksichtigen.

\begin{tabular}{|c|c|c|c|}
\hline Kategorie & Nr. & Kriterium & Beschreibung \\
\hline \multirow[t]{5}{*}{$\begin{array}{l}\text { Projekt- } \\
\text { management }\end{array}$} & A1 & $\begin{array}{l}\text { Modularisierung } \\
\text { von Projekten }\end{array}$ & $\begin{array}{l}\text { Kleine, modulare Projekte lassen sich besser führen. Große Projekte sollten in kleinere Ein- } \\
\text { heiten aufgeteilt werden. }\end{array}$ \\
\hline & $\mathrm{A} 2$ & $\begin{array}{l}\text { Definition von } \\
\text { Zielen, Scope } \\
\text { und Metrik }\end{array}$ & $\begin{array}{l}\text { Zu Beginn eines Projektes sind klare Ziele und Umfang sowie eine Metrik für die Messung } \\
\text { der Zielerreichung in einem Business Case festzulegen. }\end{array}$ \\
\hline & A3 & $\begin{array}{l}\text { Aufgaben, Rollen, } \\
\text { Verantwortlichkei- } \\
\text { ten }\end{array}$ & Aufgaben, Rollen und Verantwortlichkeiten sind zu klären. \\
\hline & A4 & $\begin{array}{l}\text { Ressourcen- } \\
\text { planung }\end{array}$ & $\begin{array}{l}\text { Die für das Projekt benötigten Ressourcen (Zeit, Personal, Kosten) sind in der Planung fest- } \\
\text { zuhalten. }\end{array}$ \\
\hline & A5 & $\begin{array}{l}\text { Monitoring und } \\
\text { Controlling }\end{array}$ & $\begin{array}{l}\text { Kontinuierliches Monitoring und Controlling des Projektes ermöglichen Korrekturmaßnah- } \\
\text { men. }\end{array}$ \\
\hline Komplexität & B1 & $\begin{array}{l}\text { Komplexität durch } \\
\text { Abhängigkeiten }\end{array}$ & $\begin{array}{l}\text { eGovernment-Projekte zeichnen sich durch eine hohe Komplexität aus. Diese resultiert aus } \\
\text { kontextbezogenen und technischen Abhängigkeiten, die u.a. aus der Größe, der Multidiszi- } \\
\text { plinarität und der Vielfalt der Akteure entsprechender Vorhaben resultieren. Die Komplexität } \\
\text { erschwert die Schätzung in der Planung und erhöht das Risiko. }\end{array}$ \\
\hline \multirow[t]{2}{*}{$\begin{array}{l}\text { Risiko- } \\
\text { management }\end{array}$} & C1 & $\begin{array}{l}\text { Identifikation } \\
\text { und Umgang mit } \\
\text { Risiken }\end{array}$ & $\begin{array}{l}\text { Die Identifizierung und der Umgang mit Risiken (vermeiden, vermindern, überwälzen, } \\
\text { akzeptieren) sowie eine Planung für den Risikofall reduzieren die Risiken bzw. ihre Folgen. }\end{array}$ \\
\hline & $\mathrm{C} 2$ & $\begin{array}{l}\text { Einsatz formaler } \\
\text { Risikomethode }\end{array}$ & $\begin{array}{l}\text { Eine formale holistische Risikomanagementmethode unterstützt diesen wiederkehrenden } \\
\text { Prozess. }\end{array}$ \\
\hline \multirow[t]{3}{*}{$\begin{array}{l}\text { Change } \\
\text { Management }\end{array}$} & D1 & $\begin{array}{l}\text { Führung von } \\
\text { Veränderungen }\end{array}$ & $\begin{array}{l}\text { Die Führung der organisationalen und kulturellen Veränderungen schafft die Vorausset- } \\
\text { zung für die Einführung und die Nutzung einer neuen Anwendung. }\end{array}$ \\
\hline & D2 & $\begin{array}{l}\text { Erwartungen und } \\
\text { Annahmen }\end{array}$ & $\begin{array}{l}\text { Der proaktive Umgang mit Erwartungen sowie realistische Annahmen über das zu realisie- } \\
\text { rende Vorhaben sind ein wichtiger Bestandteil für Veränderungen. }\end{array}$ \\
\hline & D3 & Training & $\begin{array}{l}\text { Eine effektive Nutzung der Anwendung setzt ein Training der Anwender voraus und ist } \\
\text { Bestandteil des Projektes. }\end{array}$ \\
\hline
\end{tabular}




\begin{tabular}{|c|c|c|c|}
\hline \multirow[t]{4}{*}{$\begin{array}{l}\text { Human } \\
\text { Resources }\end{array}$} & E1 & $\begin{array}{l}\text { Fachliche Kompe- } \\
\text { tenz und Erfahrun- } \\
\text { gen }\end{array}$ & $\begin{array}{l}\text { Fachliche sowie IT-Kompetenzen und Erfahrungen sind in den für das Projekt relevanten } \\
\text { Bereichen unabdingbar (Aus- und Weiterbildung). }\end{array}$ \\
\hline & E2 & $\begin{array}{l}\text { Projekt- } \\
\text { management- } \\
\text { kompetenzen }\end{array}$ & $\begin{array}{l}\text { Speziell wird dabei auf Projektmanagementkompetenzen im öffentlichen Bereich sowie } \\
\text { komplexe IT-Projekte verwiesen (Aus- und Weiterbildung). }\end{array}$ \\
\hline & E3 & $\begin{array}{l}\text { Ausreichend } \\
\text { Personal- } \\
\text { ressourcen }\end{array}$ & $\begin{array}{l}\text { Projekte sind ausreichend mit Personal zu besetzen. Vorzugsweise sollte dieses im Laufe } \\
\text { des Projektes nicht wechseln. }\end{array}$ \\
\hline & E4 & $\begin{array}{l}\text { Überschätzung } \\
\text { und Versagen }\end{array}$ & Überschätzung und personelles Versagen beeinflussen den Projektverlauf negativ. \\
\hline $\begin{array}{l}\text { Führung } \\
\text { (Leadership) }\end{array}$ & F1 & $\begin{array}{l}\text { Topmanagement- } \\
\text { Commitment }\end{array}$ & $\begin{array}{l}\text { Die Unterstützung von Projekten durch das Topmanagement (Executive Commitment) und } \\
\text { die Übertragung der Verantwortung des Erfolges auf eine Person auf Ebene Minister/Gene- } \\
\text { ralsekretär (Ownership) gibt einem Projekt die nötige Aufmerksamkeit, insb. bei knappen } \\
\text { Ressourcen. }\end{array}$ \\
\hline \multirow[t]{3}{*}{ Technologie } & G1 & $\begin{array}{l}\text { Legacy-Systeme, } \\
\text { Cutting-Edge- } \\
\text { Technologien }\end{array}$ & $\begin{array}{l}\text { eGovernment-Projekte können im seltensten Fall auf der grünen Wiese durchgeführt wer- } \\
\text { den. Entsprechend sind Legacy-Systeme zu beachten. } \\
\text { Wenn es sich nicht um den bewusst experimentellen Einsatz von Cutting-Edge-Technolo- } \\
\text { gien handelt, ist davon abzusehen. }\end{array}$ \\
\hline & G2 & IT-Sicherheit & $\begin{array}{l}\text { Speziell wird der Aspekt Sicherheit (Integrität, Verfügbarkeit und Vertraulichkeit) hervorge- } \\
\text { hoben, dem im eGovernment im Hinblick auf das Vertrauen der Anwender besondere Auf- } \\
\text { merksamkeit zu schenken ist. }\end{array}$ \\
\hline & G3 & $\begin{array}{l}\text { Adäquatheit der } \\
\text { IT-Infrastruktur } \\
\text { und Zugang }\end{array}$ & $\begin{array}{l}\text { Eine adäquate IT-Infrastruktur sowie der technische Zugang zu den eGovernment-Services } \\
\text { ist eine zentrale Voraussetzung für Betrieb bzw. Nutzung. }\end{array}$ \\
\hline $\begin{array}{l}\text { Software } \\
\text { Engineering }\end{array}$ & $\mathrm{H} 1$ & $\begin{array}{l}\text { Unterstützung } \\
\text { durch formale } \\
\text { Methoden }\end{array}$ & $\begin{array}{l}\text { Effektives Software Engineering wird durch den Einsatz formaler Methoden unterstützt. } \\
\text { Iteratives Vorgehen, Prototyping und effektives Testen spielen dabei eine wichtige Rolle. }\end{array}$ \\
\hline \multirow[t]{2}{*}{$\begin{array}{l}\text { Requirements } \\
\text { Engineering }\end{array}$} & 11 & $\begin{array}{l}\text { Verstehen der } \\
\text { Benutzer- } \\
\text { bedürfnisse }\end{array}$ & $\begin{array}{l}\text { eGovernment-Projekte müssen auf die Befriedigung echter Benutzerbedürfnisse zielen. } \\
\text { Das Nicht-Verstehen bzw. Nicht-beschreiben-Können der Benutzerbedürfnisse führt zu } \\
\text { unbefriedigenden, nicht akzeptablen Projektergebnissen. }\end{array}$ \\
\hline & 12 & $\begin{array}{l}\text { Vermeidung von } \\
\text { sich laufend } \\
\text { ändernden } \\
\text { Anforderungen }\end{array}$ & $\begin{array}{l}\text { Bei sich ständig ändernden oder unvollständigen Anforderungen ist mit einem negativen } \\
\text { Projektverlauf zu rechnen. Formale Methoden unterstützen die Erhebung der } \\
\text { Anforderungen. }\end{array}$ \\
\hline $\begin{array}{l}\text { User und } \\
\text { Stakeholder } \\
\text { Management }\end{array}$ & J1 & $\begin{array}{l}\text { Einbindung von } \\
\text { Benutzern und } \\
\text { Stakeholdern }\end{array}$ & Stakeholder und Benutzer sind frühzeitig zu involvieren. \\
\hline \multirow[t]{2}{*}{$\begin{array}{l}\text { Externe } \\
\text { Beziehungen }\end{array}$} & K1 & $\begin{array}{l}\text { Koordination und } \\
\text { Integration exter- } \\
\text { ner Partner oder } \\
\text { Lieferanten }\end{array}$ & $\begin{array}{l}\text { Im eGovernment sind verschiedene Dienstleister und Lieferanten involviert, teilweise auf- } \\
\text { grund gesetzlicher Bestimmungen. Es gilt, die externen Partner zu koordinieren, ins Projekt } \\
\text { zu integrieren und Kontakt mit dem Management seitens der Industrie zu pflegen. Eine fle- } \\
\text { xible Vertragsgestaltung erlaubt es, auf Veränderungen zu reagieren; insbesondere bei } \\
\text { Projekten mit längerer Laufzeit. }\end{array}$ \\
\hline & $\mathrm{K} 2$ & $\begin{array}{l}\text { Anzahl und Repu- } \\
\text { tation der Partner }\end{array}$ & $\begin{array}{l}\text { Die Anzahl der Partner sowie deren Reputation und Zuverlässigkeit sind weitere } \\
\text { Subfaktoren. }\end{array}$ \\
\hline $\begin{array}{l}\text { Finanzen und } \\
\text { Wertbeitrag }\end{array}$ & L1 & $\begin{array}{l}\text { Finanzierung vs. } \\
\text { Sparmaßnahmen; } \\
\text { Berücksichtigung } \\
\text { über den ganzen } \\
\text { IT-Lifecycle }\end{array}$ & $\begin{array}{l}\text { Die Finanzierung sollte auf einer soliden Basis stehen. Im Verwaltungsumfeld können Spar- } \\
\text { maßnahmen Projekte kurzfristig beenden oder unterbrechen. Ein eindeutiger Gesetzesauf- } \\
\text { trag schränkt diese Option ein. Im Sinne des Value for Money bzw. der Total Cost of } \\
\text { Ownership (TCO) müssen Nutzen und Kosten des gesamten Lifecycles betrachtet werden. }\end{array}$ \\
\hline $\begin{array}{l}\text { Rechtliche } \\
\text { Rahmen- } \\
\text { bedingungen }\end{array}$ & M1 & $\begin{array}{l}\text { Berücksichtigung } \\
\text { des Legalitäts- } \\
\text { prinzips und des } \\
\text { Datenschutzes }\end{array}$ & $\begin{array}{l}\text { Das Handeln der Verwaltung ist an Rechtsvorschriften gebunden. Die Verwaltung kann nur } \\
\text { in diesem Rahmen tätig werden bzw. hat diese zu beachten. Dies hat u.a. Einfluss auf die } \\
\text { Umsetzung von Projekten, beispielsweise bei öffentlichen Ausschreibungen. Daher ist es } \\
\text { unabdingbar, rechtliche Grundlagen zu schaffen oder bestehende Rechtsvorschriften anzu- } \\
\text { passen. Der Datenschutz ist diesem Faktor zugehörig. }\end{array}$ \\
\hline \multirow[t]{2}{*}{ Organisation } & N1 & $\begin{array}{l}\text { IT-Governance } \\
\text { und über- } \\
\text { geordnete } \\
\text { Organisationsziele }\end{array}$ & $\begin{array}{l}\text { Eine Vision und eine davon abgeleitete Strategie geben die Rahmenbedingungen und glo- } \\
\text { balen Ziele für die Entwicklung von eGovernment vor; dies im Sinne der IT-Governance. } \\
\text { Konkrete Vorhaben leisten einen Beitrag zu den Organisationszielen. }\end{array}$ \\
\hline & N2 & $\begin{array}{l}\text { Anzahl involvierter } \\
\text { OE }\end{array}$ & $\begin{array}{l}\text { Die Anzahl involvierter Organisationseinheiten (OE), das Verhältnis derselben zueinander } \\
\text { sowie die um Ressourcen konkurrierenden Projekte sind weitere Subfaktoren. }\end{array}$ \\
\hline $\begin{array}{l}\text { Kontext und } \\
\text { Umwelt }\end{array}$ & $\mathrm{O} 1$ & $\begin{array}{l}\text { Kontext und } \\
\text { Umwelt }\end{array}$ & $\begin{array}{l}\text { Ein Projekt spielt sich in einem Kontext ab, der maßgeblich von der externen Umwelt } \\
\text { (z.B. Politik, Gesellschaft) und der internen Projektumwelt (Verwaltung, Personal etc.) } \\
\text { beeinflusst wird. }\end{array}$ \\
\hline
\end{tabular}

Tab. 2: Synopse der IT-Erfolgsfaktoren 
Abhängig von der Betrachtungsperspektive können unterschiedliche Bündel von Erfolgsfaktoren für das IT-Management wesentlich sein. Als Betrachtungsperspektiven können beispielhaft genannt werden: strategisches vs. operatives IT-Management sowie die operativen Prozessphasen im IT-Lifecycle. Ferner sollten unterschiedliche Projekttypen differenziert betrachtet werden. Beispielsweise weist ein strategisches Architekturprojekt andere Erfolgsfaktorenbündel auf (etwa Organisation, Komplexität, Umwelt) als ein Projekt zur Entwicklung einer Fachapplikation (etwa Software Engineering und Requirements Engineering). Dies impliziert unterschiedliche Risikoaspekte oder -adressierungen in unterschiedlichen Vorhaben. Für die abgeleitete Risikobetrachtung können Entscheide im IT-Management unterschiedliche Folgen haben, etwa was Make-or-buy-, Prozessoptimierungs- oder Software- und Hardwareentscheide betrifft. Zudem sind die Erfolgsfaktoren je nach Betrachtungsperspektive unterschiedlich. Dies bedeutet, dass eine Rangreihung der Erfolgsfaktoren problematisch ist. Es lässt sich jedoch durch eine Häufung der Nennungen von Erfolgsfaktoren - mit Vorbehalten - eine generische Tendenz ableiten.

\section{Einsatz von IT-Governance- Methoden in der schweizerischen Bundesverwaltung}

In der schweizerischen Bundesverwaltung (BV) kommen - ausgehend vom Projekt NOVE-IT, das der Neuorganisation der IT gewidmet war-verschiedene IT-Governance-Methoden zum Einsatz, die zwingend einzusetzen sind. Diese sind in der Folge kurz beschrieben.

\subsection{Beschreibung der IT-Governance- Methoden}

HERMES ist die Standard-Projektführungsmethode, die in der Bundesverwaltung entwickelt wurde und eingesetzt wird. Sie ist in die zwei
Teile Systementwicklung und Systemadaption [HERMES 2003 u. 2005] aufgeteilt. Für die Prüfung von IT-Projekten setzt die Eidgenössische Finanzkontrolle (EFK) u.a. für die Ex-post-Analyse der IT-Organisation und der IT-Governance CobiT 4.0 ein. Die ICO-Methode [ICO-Methode 2007] wird in der Bundesverwaltung als Standard für das finanzielle und qualitative IT-Controlling und das IT-Portfoliomanagement angewendet. Neben dem bundeseigenen IT-Prozessmodell, das Leistungserbringer und -bezüger einschließt, wird auf der Leistungserbringerseite im Betrieb partiell ITIL V2 eingesetzt. Die vorgestellten Methoden stehen in einem bestimmten Verhältnis zueinander, was für das Mapping mit den Erfolgsfaktoren relevant ist. Die Methoden decken damit aus unterschiedlichen organisatorischen Perspektiven jeweils partielle Bereiche eines umfassenden IT-Managements ab, teilweise unter Einbezug von Aspekten des IT-Risikomanagements.

\subsection{Vergleich der Methoden in Analogie zu den Erfolgsfaktoren}

Im rechten Teil der Tabelle 3 wird in Form einer Matrix untersucht, in welchen Methoden eine Übereinstimmung mit den Erfolgsfaktoren besteht. Als Resultat soll ein etwaiger Nachholbedarf bezüglich IT-Risikomanagement in der Bundesverwaltung oder im eGovernment eruiert werden. Im Falle von CobiT 4.0 wird wo möglich die Prozess- oder Prüfgruppe angegeben, unter der die entsprechenden Kategorien der Erfolgsfaktoren abgehandelt werden. Es kann festgestellt werden, dass die Abdeckung in CobiT 4.0 verhältnismäßig groß ist und dass auch in HERMES diverse Aspekte abgehandelt werden, die aus Sicht der Erfolgsfaktoren ins Gewicht fallen. Die IT-Controlling-Methode ICO hat ihrer Natur nach eher geringe Überschneidungen mit den entsprechenden Erfolgsfaktoren in Projekten.

Bei aller Nachforschung konnte jedoch aus Sicht des Risikomanagements keine integrierte und holistische Methode zur systematischen 
Verringerung des IT-Risikos in der BV gefunden werden. Risikoaspekte sind zudem über die Erfolgsfaktoren hinaus unterschiedlich marginal in den entsprechenden Methoden und Rahmenwerken enthalten. Der Projektführungsmethode HERMES kann zugute gehalten werden, dass die Eingrenzung des Projektes im Sinne der Anpassung der Methode und der entsprechend zu erstellenden Unterlagen mustergültig ist. Der Verdacht lässt sich aber nicht von der Hand weisen, dass statische IT-Risikodokumentation als proaktives IT-Risikomanagement getätigt wird. Da ITIL derzeit eher im IT-Betrieb eingesetzt wird, ist die Übereinstimmung bezüglich der Erfolgsfaktoren in Projekten gering. Dies dürfte sich mit dem Übergang zu ITIL $V_{3}$ ändern. Zudem erweisen sich die Charakteristika der einzelnen erwähnten Methoden als Nach- teile. So steht bei HERMES das Projektmanagement im Vordergrund und nicht das integrale IT-Management (im Sinne des Plan-Build-Run). Bei CobiT steht meist ex-post das Auditing von IT-Vorhaben im Vordergrund. ITIL deckt nur den Betriebsaspekt der IT ab. Bei der ICO-Methode stehen auf Basis entsprechender ControllingBerichte schwergewichtig finanzielle und qualitative Aspekte im Vordergrund. Gesamthaft ist anzumerken, dass eher holistisch zu betrachtende Risikoaspekte beim "Puzzle« der verschiedenen IT-Governance-Methoden in Relation zur IT-Organisation zu einem Problem werden (können). Insbesondere wäre ein integriertes Risikoinstrumentarium wünschenswert, das über den gesamten IT-Lifecycle Anwendung findet.

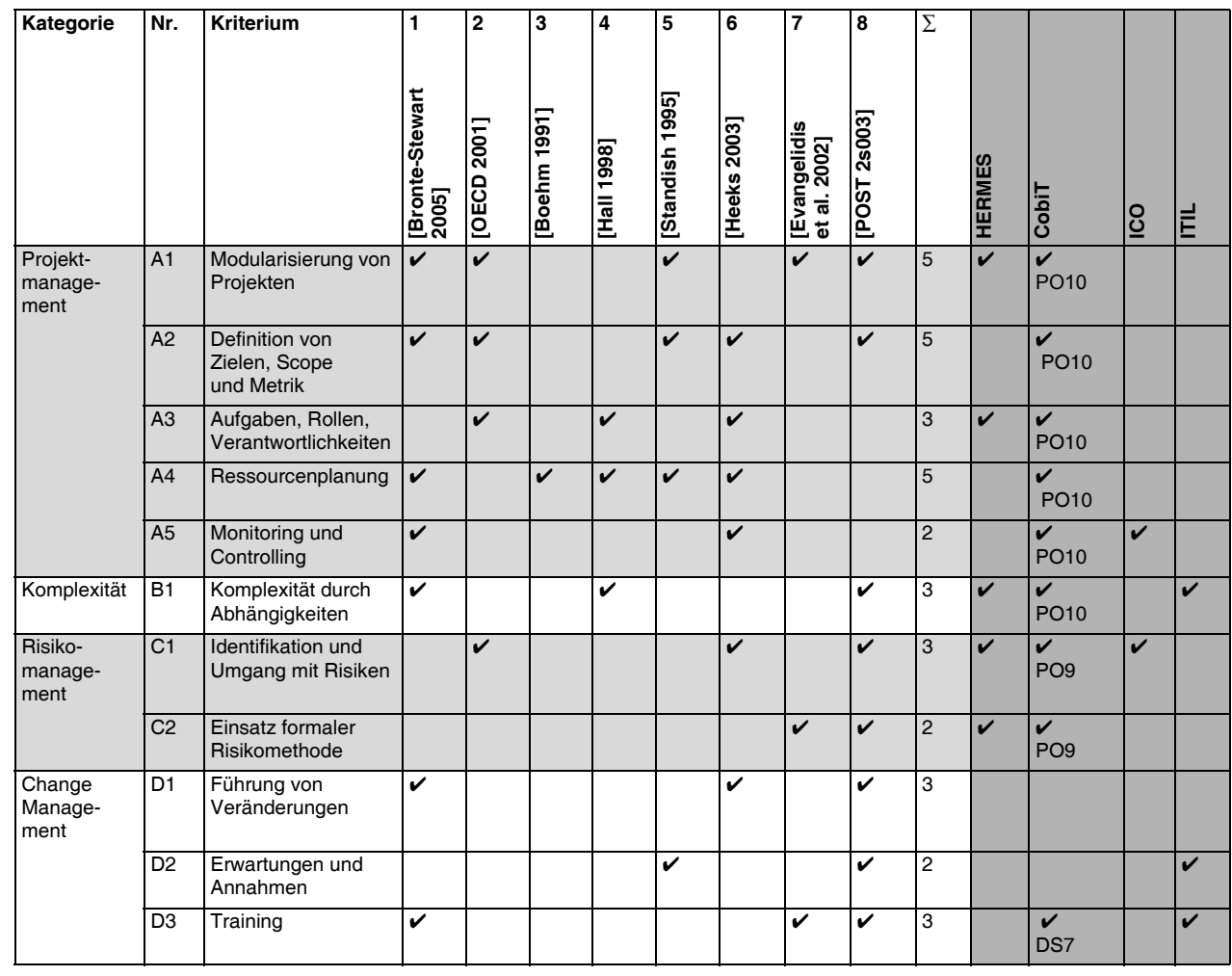




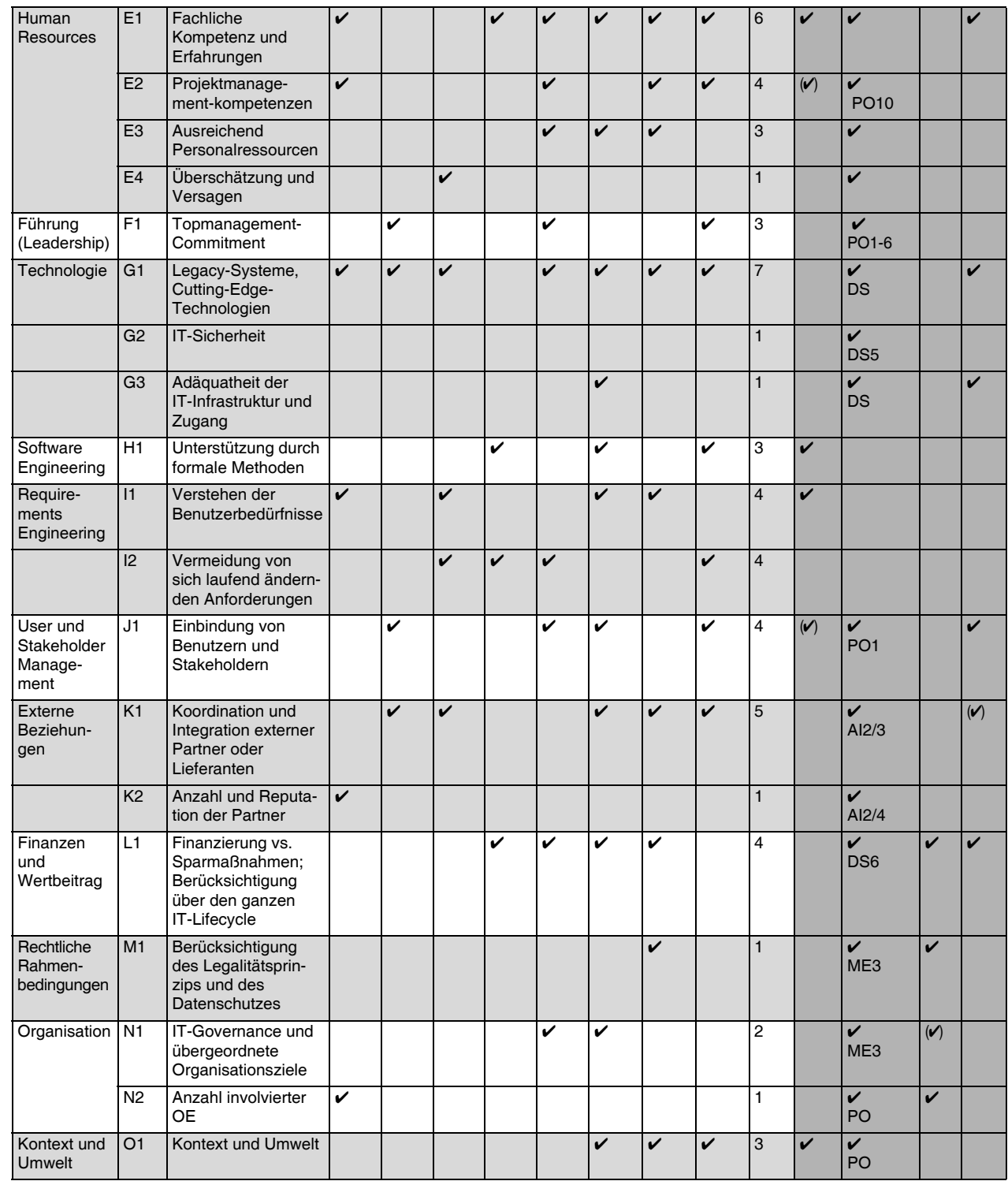

Tab. 3: IT-Erfolgsfaktoren und deren Abdeckung in den IT-Governance-Methoden für Projekte

\section{Herausforderungen für das IT-Risikomanagement}

Die Gegenüberstellung der Erfolgsfaktoren und der in der BV eingesetzten IT-Governance-Methoden ergab eine partielle Deckung. Dies lässt sich daraus ableiten, dass IT-Governance-Metho- den jeweils separate Teilbereiche des IT-Managements aus unterschiedlichen organisatorischen Perspektiven abdecken. Damit bleiben holistische Aspekte sowie kausale Zusammenhänge zwischen verschiedenen Risikofaktoren möglicherweise unberücksichtigt. Entsprechend sollten nicht abgedeckte Risikoaspekte mit separa- 
ten und allenfalls umfassenderen Methoden berücksichtigt werden. Zu einem vergleichbaren Ergebnis im eBusiness kommen [Junginger \& Krcmar 2001], die eine "Führungslücke» in den Konzepten und Methoden des IT-Risikomanagements eruieren. Hinsichtlich einer weiter gehenden Abdeckung über die IT-Governance-Methoden hinaus sind Fragen über die organisationale Implementierung des IT-Risikomanagements im Hinblick auf Aufgaben, Kompetenzen und Verantwortlichkeiten zu adressieren.

Aus Sicht des Versuchs, eine Synopse der ITErfolgsfaktoren zu erstellen, lässt sich erstens kritisieren, dass die untersuchten Studien hauptsächlich Projekte in den Vordergrund der Betrachtung stellten und dabei nur begrenzt Aussagen über ein übergeordnetes IT-Management zulassen. Zweitens werden verschiedene aus Sicht der Verfasser wichtige Kriterien für das Risikomanagement im IT-Bereich nicht erwähnt und sind daher vertiefter zu diskutieren. Dies schließt insbesondere Risiken ein, die über die IT-Organisation hinausgehen. Nicht zuletzt müssen Erfolgsfaktoren aus unterschiedlichen Kontexten auf ihre Transferierbarkeit und ihren Nutzen für die Praxis hinterfragt werden. Dennoch kann auf konzeptioneller Ebene von einer Allgemeingültigkeit der Resultate im Hinblick auf die Risiken in eGovernment-Projekten und den IT-Governance-Methoden ausgegangen werden. Auf konzeptioneller Ebene dürften die Herausforderungen komplexer IT-Projekte in unterschiedlichen nationalen eGovernmentKontexten ähnlich sein. Dies gilt insbesondere auch für unterschiedliche IT-Governance-Methoden, die sich, wenn nicht bereits ein internationaler Standard verwendet wird, zumindest an einen solchen Standard anlehnen. Jedoch können in der konkreten Umsetzung erhebliche Unterschiede auftreten, etwa abhängig davon, wie eine Verwaltungsorganisation aufgebaut ist (föderalistisch oder zentralistisch) oder welche Maturität im Umgang mit komplexen und tendenziell risikobehafteten Projekten eine Organisation hat.
In einem strukturierten Interview mit einem Mitglied der Eidgenössischen Finanzkontrolle EFK hat sich zudem gezeigt, dass einem erweiterten IT-Risikomanagement ein Nutzen attestiert würde. Jedoch sind die entsprechenden personellen Ressourcen für die laufende vertiefte Prüfung von Risikoaspekten im IT-Management in der schweizerischen Bundesverwaltung zu gering. Neben dem Risikomanagement für Projekte sollen auch das Risikomanagement für das strategische IT-Management, für Vorstudien zu Projekten und zum Betrieb vertieft werden. Weiter und differenzierter zu betrachten sind: organisationales Change Management, IT-Führung, externe IT-Beziehungen und IT-Organisation. Eine Verstärkung ist somit an den Stellen erforderlich, wo es um die integrale Ex-ante-Berücksichtigung von Erfolgsoder Risikofaktoren im IT-Management geht. Beim IT-Management konnten Lücken in der Abdeckung festgestellt werden. In diesem Sinne sollte es sich bei den zusätzlich zu berücksichtigenden Methoden des Risikomanagements primär um Ex-ante- und begleitende Maßnahmen bzw. Evaluationen handeln, die ressourceneffizient anwendbar sind. Die Möglichkeit, in diesem Bereich weitere Forschungsergebnisse zu liefern, dürfte nicht nur für das ITManagement im eGovernment, sondern auch generell für die Betrachtung des IT-Risikomanagements von Interesse sein. In einem weiteren Schritt eines explorativen Forschungsvorgehens wird in Form von Fallstudien, d.h. zunächst mittels qualitativer Forschung, den Gründen des Scheiterns von eGovernment-Projekten in der BV nachgegangen. Dabei sollen die Erfolgsfaktoren validiert, gruppiert, bewertet und wo möglich durch weiche Faktoren ergänzt werden. Ein längerfristiges Forschungsziel ist darauf ausgerichtet, eine integrale Auditmethode für das Risikomanagement im eGovernment zu erarbeiten. 


\section{Literatur}

[Boehm 1991] Boehm, B. W.: Software Risk Management: Principles and Practices. IEEE Software, 8 (1), 1991.

[Bronte-Stewart 2005] Bronte-Stewart, M.: Developing a risk estimation model from IT project failure research. Computing and Information Systems Journal, 9 (3), 2005.

[Evangelidis et al. 2002] Evangelidis, A. et al.: Risk Assessment \& Success Factors for e-Government in a UK Establishment. In: EGOV '02: Proceedings of the First International Conference on Electronic Government, 2002.

[Hall 1998] Hall, E.: Managing risk: methods for software systems development. Reading, MA, 1998.

[Heeks 2003] Heeks, R.: eGovernment for Development: Causes of eGovernment Success and Failure: Factor Model, IDPM, 2003, www.egov4dev.org/causefactor.htm; Zugriff am 27.09.2007.

[HERMES 2003] HERMES: Systementwicklung. ISB, Bern, 2003.

[HERMES 2005] HERMES: Systemadaption. ISB, Bern, 2005.

[ICO-Methode 2007] ICO-Methode: Informatikcontrolling ICO, Version 4.0, EFD, 2007.

[Junginger \& Krcmar 2001] Junginger, M.; Krcmar, H.: IT-Risk Management - Fit für E-Business? In: Buhl, H. U.; Huther, A.; Reitwieser, B.: Information Age Economy. 5. Internationale Tagung Wirtschaftsinformatik 2001, Heidelberg, 2001.

[Mertens 2008] Mertens, P.: Fehlschläge bei ITGroßprojekten der Öffentlichen Verwaltung ein Beitrag zur Misserfolgsforschung in der Wirtschaftsinformatik. In: Bichler, M.; Hess, T.; Krcmar, H.; Lechner, U.; Matthes, F.; Picot, A.; Speitkamp, B.; Wolf, P. (Hrsg.): Tagungsband zur Multikonferenz Wirtschaftsinformatik München, GITO-Verlag, Berlin, 2008, S. 243-244.
[Moll 1994] Moll, K.-R.: Informatik-Management Aufgabengebiete, Lösungswege, Controlling. Springer-Verlag, Berlin et al., 1994.

[OECD 2001] OECD: The hidden threat to E-Government - Avoiding large government IT failures. OECD Public Management Policy Brief No. 8, 2001.

[Peterson \& Kim 2000] Peterson, D. K.; Kim, C. S.: Information system objectives: effects of experience, position level and education on developers. Journal of Information Technology Management, Vol. 11, 2000.

[POST 2003] POST: Government IT projects. Parliamentary Office of Science and Technology, Report 200, 2003.

[Standish 1995] The Standish Group International: CHAOS Report. The Standish Group International, Inc., 1995.

[Wallmüller 2004] Wallmüller, E.: Risikomanagement für IT- und Software-Projekte. Hanser Verlag, München, 2004.

Dipl.-Wirtsch.-Inf. Andreas Kühn

Dr. Konrad Walser

Prof. Dr. Reinhard Riedl

Kompetenzzentrum Public

Management und E-Government

Berner Fachhochschule

Morgartenstr. $2 a$

$\mathrm{CH}-3000$ Bern 22

\{andreas.kuehn, konrad.walser, reinhard.riedl@bfh.ch\}

www.e-government.bfh.ch 